\title{
Anticancer Effect of ERM210 on Liver Cancer Cells Through ROS/Mitochondria-dependent Apoptosis Signaling Pathways
}

\author{
JAIHYUNG LEE ${ }^{1 *}$, YI-XI GONG ${ }^{2 *}$, DAN-PING XIE ${ }^{2 *}$, HYUNJEONG JEONG $^{1}$, \\ HOYOUNG SEO ${ }^{1}$, JIHWAN KIM $^{3}$, YANG HO PARK ${ }^{4}$, HU-NAN SUN ${ }^{2}$ and TAEHO KWON ${ }^{5}$ \\ ${ }^{1}$ Epigenetics Drug Discovery Center, Haeam Convalescence Hospital, Gyeonggi, Republic of Korea; \\ ${ }^{2}$ College of Life Science \& Biotechnology, Heilongjiang Bayi Agricultural University, Daqing, P.R. China; \\ ${ }^{3}$ Korean Convergence Medicine Center, 100 years Oriental Medical Clinic, Seoul, Republic of Korea; \\ ${ }^{4}$ Evidence-based Medicine Center, Park Yang Ho BRM Institute, Seoul, Republic of Korea; \\ ${ }^{5}$ Primate Resources Center, Korea Research Institute of Bioscience and Biotechnology (KRIBB), \\ Jeonbuk, Republic of Korea
}

\begin{abstract}
Background/Aim: Asian Traditional medicines are renowned for their antitumor properties and are efficacious in the clinical treatment of various cancer types. ERM210 is a Korean traditional medicine comprising nine types of medicinal plants. In the present study, we examined the pro-apoptotic effect and molecular mechanisms of the effects of ERM210 on HepG2 liver cancer cells. Materials and Methods: The cytotoxicity of ERM210 on HepG2 cells was investigated using 3-(4,5-dimethylthiazol-2-yl)-2,5diphenyltetrazolium bromide and wound-healing assays, and apoptosis and signaling pathways by fluorescence microscopy flow cytometry and western blotting. Results: ERM210 significantly impaired HepG2 cell viability and enhanced mitochondria-dependent cellular apoptosis in a time- and dose-dependent manner by up-regulating the expression of caspases 3, 7 and 9, and of BCL2 apoptosis regulator (BCL2)-associated $X$, apoptosis regulator $(B A X)$ proteins, whilst down-regulating that of BCL2 protein. Furthermore, ERM210 treatment increased accumulation of cellular and mitochondrial reactive oxygen species (ROS)
\end{abstract}

This article is freely accessible online.

*These Authors contributed equally to this study.

Correspondence to: Dr. Taeho Kwon, Primate Resources Center, Korea Research Institute of Bioscience and Biotechnology, 351-33 Neongme-gil, Ibam-myeon, Jeongeup-si, Jeonbuk 56216, Republic of Korea. E-mail: kwon@kribb.re.kr; Dr Hu-Nan Sun, College of Life Science \& Technology, Heilongjiang Bayi Agricultural University, \#2 Xinyanglu, 163319, Daqing, P.R. China. E-mail: sunhunan76@163.com

Key Words: Anticancer, apoptosis, ERM210, mitochondria, reactive oxygen species. and significantly inhibited cell migration. Additionally, all these phenomena were reversed by treating with the ROS scavenger $N$-acetylcysteine. The analysis of signaling proteins revealed that ERM210 significantly up-regulated the phosphorylation of ROS-dependent mitogen-activated protein kinases (p38, extracellular-regulated kinase, and c-Jun $\mathrm{N}$ terminal kinase in HepG2 liver cancer cells. Conclusion: ERM210 exerts anticancer effects in HepG2 liver cancer cells by up-regulating ROS/mitochondria-dependent apoptosis signaling, providing new insight into the possibility of employing this traditional medicine for the clinical treatment of liver cancer.

A wide variety of unique traditional herbal medicines abound in Asia. These medicines have numerous advantages over conventional chemotherapy drugs, such as low toxicity, easy absorption, and multi-target action $(1,2)$. According to the World Health Organization, traditional medicine is "used in the maintenance of health as well as in the prevention, diagnosis, improvement or treatment of physical and mental illness" (3). This underlies the long-established traditional use of herbal-based medicines. It includes herbal medicines and herbal finished products, which contain plants and other combination as active ingredients (4). In recent years, numerous studies have shown that herbal medicine is effective in treating insomnia (3), Alzheimer's disease (5), cardiovascular diseases (6), polycystic ovary syndrome (7), esophageal cancer (8), and other diseases (9, 10). Herbal medicines contain abundant anticancer compounds that directly affect cancer cells and have been utilized as anticancer agents far longer than any of the chemotherapy drugs.

Cancer is an intractable human disease. Liver cancer is for the third leading cause of mortality (11). Hepatocellular carcinoma constitutes the primary and most common liver cancer subtype, with contributing risk factors including 
chronic viral hepatitis B and C, aflatoxin exposure, alcohol, and non-alcohol fatty liver disease or steatohepatitis. Surgical resection, radiotherapy, and chemotherapy comprise the commonly applied treatment methods for afflicted patients. However, surgical resection is more effective for patients diagnosed in the preliminary stages of the disease. For patients with highly invasive and metastatic advancedstage hepatocellular carcinoma, chemotherapy remains the most viable option. Although remarkable milestones and considerable progress have been achieved in cancer therapeutics, first-line anticancer drugs, such as sorafenib, 5fluorouracil, and cisplatin, produce highly toxic side-effects in patients receiving them. These include vomiting, dizziness, and hair loss. This necessitates the development of novel anticancer drugs.

Reactive oxygen species (ROS) are molecules that are not only associated with deleterious effects but also act as signaling molecules in multiple cellular signaling pathways that decide the fate of both normal cells and tumor cells. Research shows that ROS exert multifunctional regulatory actions over proteins, nucleic acids, lipids, and other biological macromolecules. When present in excess, they adversely affect the normal physiology of the cell (12-14). ROS have been implicated in tumor initiation, tumor progression, and metastasis (15). It is established that the mitochondria play multiple essential roles in intracellular ROS generation (16). However, the ROS level in tumor cells was found to be higher than those in normal cells due to their enhanced glucose metabolism, leading to mitochondrial dysfunction and oncogenic activity (17). The resulting oxidative stress may also generate potential antitumor effects. Oxidative stress can cause damage to mitochondria that is disruptive to normal function, including significantly reducing the mitochondrial membrane potential and elevating the ROS level in mitochondria, thereby inducing cell death.

Mitogen-activated protein kinase (MAPK) signaling is an important signal transduction pathway in cells, and is primarily involved in the regulation of apoptosis, cell metabolism, cell differentiation and tumorigenesis, in addition to other essential life processes $(18,19)$. MAPKs are an extensive family of protein kinases, including three main subfamilies: Extracellular signal-regulated kinase (ERK), c-Jun $N$-terminal kinase (JNK), and P38 MAPK. The MAPK family plays a crucial role in ROS generationinduced apoptosis (20).

An increasing number of clinical studies have reported that Asian traditional herbal medicine can relieve disease symptoms, improve the quality of life, and prolong the survival of patients with cancer. Some Asian traditional herbal medicines are considered to be uniquely advantageous in the treatment of certain tumors (21); however, their underlying mechanisms of action remain elusive. Numerous active compounds in Asian traditional herbal medicine exert
Table I. The composition of ERM210 by dry weight.

\begin{tabular}{lccr}
\hline Latin name & Country of origin & Amount (g) & $\%$ \\
\hline $\begin{array}{l}\text { Picrasma quassioides } \\
\text { (D. Don) Benn }\end{array}$ & Korea & 6.0 & 19 \\
Polygonum cuspidatum & Korea & 4.0 & 13 \\
$\begin{array}{l}\text { Sophora flavescens } \\
\text { Solander ex Aiton }\end{array}$ & Korea & 2.5 & 8 \\
Gossypium indicum Lamarck & Korea & 3.5 & 11 \\
Picrorhiza kurroa Bentham & Korea & 3.5 & 11 \\
Chelidonium majus Linne & Korea & 3.0 & 10 \\
Cinnamomum cassia & Korea & 3.0 & 10 \\
Achyranthes bidentata & Korea & 5.0 & 16 \\
Zingiber officinale Rosc & Korea & 0.5 & 2 \\
Total & & 31 & 100 \\
\hline
\end{tabular}

a variety of antitumor effects, likely through the enhancement of the ROS level in treating patients with cancer $(22,23)$. Therefore, the suitable manipulation of ROS as anticancer agents is immensely challenging.

ERM210 is a formulation of extracts from nine Asian plants (Picrasma quassioides (D. Don) Benn, Polygonum cuspidatum, Sophora flavescens Solander ex Aiton, Gossypium indicum Lamarck, Picrorhiza kurroa Bentham, Chelidonium majus Linne, Cinnamomum cassia, Achyranthes bidentata, Zingiber officinale Rosc). In the present study, we demonstrated that the MAPK signaling pathway is significantly altered during ERM210-induced apoptosis of HepG2 cells.

\section{Materials and Methods}

ERM210 formulation. ERM210 is composed of nine oriental medicinal herbs (Table I). The batch used in the present study comprised the following by dry weight: $19 \%$ Picrasma quassioides (D. Don) Benn (Korea), 13\% Polygonum cuspidatum (Korea), 8\% S. flavescens Solander ex Aiton (Korea), $11 \%$ G. indicum Lamarck (Korea), 11\% Picrorhiza kurroa Bentham (Korea), 10\% Chelidonium majus Linne (Korea), $10 \%$ Cinnamomum cassia (Korea), 5\% A. bidentata (Korea), and 2\% Z. officinale Rosc (Korea). The herbal components were obtained from the Haeam Convalescence Hospital (Gapyeong-gun, Gyeonggi-do, Republic of Korea) and kindly authenticated by Dr. Jaihyung Lee, KMD, Ph.D. (Haeam Convalescence Hospital, Republic of Korea).

Extraction of ERM210 from the constituent herbs. The extract was prepared as follows: The dried and pulverized medicinal herbs were mixed and batches weighing $31 \mathrm{~g}$ each were soaked in distilled water and shaken at $37^{\circ} \mathrm{C}$ at a speed of $200 \mathrm{rpm} / \mathrm{min}(5 \mathrm{l})$. The extract was concentrated with a rotary evaporator, lyophilized and $5 \mathrm{~g}$ extract was obtained which was reconstituted in $1 \times$ Dulbecco's modified Eagle's medium (DMEM) for the in vitro studies.

Cell culture. The HepG2 hepatoblastoma cell line (ATCC ${ }^{\circledR} \mathrm{HB}-$ 8065; American Type Culture Collection Manassas, VA, USA) and 
QSG-7701 non-transformed hepatocyte cell lines (ATCC ${ }^{\circledR}$ CL-0419; American Type Culture Collection) were cultured in DMEM (Invitrogen, Carlsbad, CA, USA) containing 10\% (v/v) fetal bovine serum (Hyclone, Logan, UT, USA), and $100 \mathrm{U} / \mathrm{ml}$ penicillin and $100 \mathrm{mg} / \mathrm{ml}$ streptomycin (Solarbio Life Sciences, Beijing, P.R. China) at $37^{\circ} \mathrm{C}$ in a humidified incubator under $5 \% \mathrm{CO}_{2}$.

Cell viability assay. The cell viability was analyzed with the 3-(4,5dimethylthiazol-2-yl)-2,5-diphenyltetrazolium bromide (MTT; Sigma-Aldrich, St. Louis, MO, USA) assay. Both HepG2 and QSG7701 cells were seeded into 96 -well plates at a density of $5 \times 10^{5}$ cells $/ \mathrm{ml}$. After culturing in DMEM for $24 \mathrm{~h}$, the cells were treated for $24 \mathrm{~h}$ with ERM210 at different concentrations $(0,5,10,15$, and $20 \%$; drug concentration being $5 \% \mathrm{v} / \mathrm{v}$ of DMEM). Thereafter, 10 $\mu \mathrm{l}(0.5 \mathrm{mg} / \mathrm{ml})$ of MTT solution was added into each well of the 96well plates and cells were incubated for a further $2 \mathrm{~h}$ at $37^{\circ} \mathrm{C}$ under $5 \% \mathrm{CO}_{2}$. Subsequently, the supernatant was removed, and formazan was solubilized in $100 \mu \mathrm{l}$ dimethyl sulfoxide. The absorbance was measured at $490 \mathrm{~nm}$ using a UV MAX kinetic microplate reader (Molecular Devices, LLC, Sunnyvale, CA, USA).

Apoptosis detection. Flow cytometry was used to detect cell apoptosis with ERM210 at concentrations of $0,10,15$, and $20 \%$. HepG2 cells were seeded into 6-well plates at a density of $9 \times 10^{4}$ cells $/ \mathrm{ml}$, cultured for $12 \mathrm{~h}$, and then treated with ERM210 at concentrations of $0,10,15$, and $20 \%$ for $24 \mathrm{~h}$, then cells were stained using annexin V-fluorescein isothiocyanate/propidium iodide (Solarbio Life Sciences, Beijing, PR China), according to the manufacturer's protocol. Cells were recovered and analyzed by flow cytometry (FACSCalibur, BD Biosciences, Franklin Lakes, NJ, USA). The results were analyzed using WinMDI software (version 2. 9; BD Biosciences).

Cellular ROS detection. HepG2 cells were seeded in 6-well plates, at a density of $10^{5}$ cells $/ \mathrm{ml}$, and then pre-treated with the ROS scavenger $N$-acetylcysteine (NAC; $5 \mu \mathrm{M}$ ) for $30 \mathrm{~min}$ or treated with ERM210 at concentrations of $0,10,15$, and $20 \%$. This was followed by incubation for $24 \mathrm{~h}$. To evaluate the changes in HepG2 cellular ROS levels, the cells were processed using $1 \mu \mathrm{M}$ dihydroethidium (DHE; Beyotime Biotechnology, Shanghai, PR China) and MitoSOX (Thermo Fisher Scientific, Waltham, MA, USA) staining. Cell nuclei were visualized using Hoechst 32258 (Thermo Fisher Scientific) staining $(2 \mu \mathrm{g} / \mathrm{ml})$ for a 20 -min incubation period. Subsequently, the ROS levels in different treatment groups were qualitatively assessed under a microscope.

Wound-healing assay. Cells treated with/without ERM210, alone and pretreated with $5 \mu \mathrm{M} \mathrm{NAC}$ for $30 \mathrm{~min}$, were seeded into 12well plates at a density of $25 \times 10^{4}$ cells/well and then cultured in DMEM for $12 \mathrm{~h}$. Subsequently, linear wounds of equal width were created using $10 \mu \mathrm{l}$ pipette tips. The culture medium was changed to that DMEM containing $1 \%$ fetal bovine serum to be cultured for another $24 \mathrm{~h}$. The cells were observed and images were captured at $0 \mathrm{~h}$ and $24 \mathrm{~h}$ using fluorescence microscopy.

Western blot assay. HepG2 cells were treated with 15\% ERM210 for $0,1,3,6,12$, and $24 \mathrm{~h}$, following which the cells were recovered and lysed. Subsequently, the cell protein lysates were separated on $12 \%$ sodium dodecyl sulfate-polyacrylamide gels and transferred onto nitrocellulose membranes (Millipore, Bedford, MA,
USA). The membranes were incubated with primary antibodies against vimentin (\#sc-373717; Santa Cruz Biotechnology, Dallas, TX, USA), p-P38 (\#sc-7973; Santa Cruz Biotechnology), P38 (\#LFMA0126; AbFrontier, Seoul, Republic of Korea), p-ERK (\#sc-7383; Santa Cruz Biotechnology), ERK (\#sc-135900; Santa Cruz Biotechnology), p-JNK (\#sc-6254; Santa Cruz Biotechnology), JNK (\#sc-7345; Santa Cruz Biotechnology), BCL2 apoptosis regulator (BCL2; \#sc-7382; Santa Cruz Biotechnology), BCL2-associated X, apoptosis regulator (BAX; \#ab90435; Abcam Cambridge, MA, USA), cleaved caspase-9 (\#9505; Cell Signaling Technology, Beverly, MA, USA), cleaved caspase-7 (\#sc-56063; Santa Cruz Biotechnology), cleaved caspase-3 (\#9661; Cell Signaling Technology, Beverly, MA, USA), $\beta$-actin (\#ab7291, dilution 1:2,000; Abcam) at $4^{\circ} \mathrm{C}$ for $12 \mathrm{~h}$. The membranes were washed five times with tris-buffered saline containing Tween-20 [TBST;10 mM Tris $\mathrm{HCl}(\mathrm{pH} 7.5), 150 \mathrm{mM} \mathrm{NaCl}$, and $0.2 \%$ Tween-20] and subsequently incubated with horseradish peroxidase-conjugated goat anti-rabbit IgG or anti-mouse IgG for $1 \mathrm{~h}$ at room temperature. Following the removal of excess antibodies by washing with TBST, specific binding was detected using a chemiluminescence detection system (GE Healthcare Life Sciences, Little Chalfont, UK) according to the manufacturer's protocol.

Statistical analysis. All the data from at least three independent experiments are reported as the mean \pm standard deviation. Repeated measures of the two-way analysis of variance were used to analyze the period and differences between groups in each experiment. Differences between groups in each experiment with values of $p<0.05$ were considered indicative of significant difference.

\section{Results}

ERM210 reduced cell viability by inducing apoptosis of HepG2 liver cancer cells. To investigate the cytotoxic effects of ERM210 on liver cells, HepG2 liver cancer cells and QSG-7701 normal liver cells were treated with ERM210 at different concentrations (0-20\%) for $24 \mathrm{~h}$. Cell viability was then detected using MTT assay as described in the Materials and Methods. The obtained results showed that ERM210 treatment significantly reduced HepG2 liver cancer cell viability at concentrations of $15 \%$ and $20 \%$ while generating weak cytotoxicity in QSG-7701 normal liver cells (Figure 1A). To clarify the effects of ERM210 on cell apoptosis, HepG2 cells were treated with ERM210 at various concentrations (0-20\%) for $24 \mathrm{~h}$, following which the apoptosis of HepG2 cells was detected by flow cytometry. As shown in Figure 1B and C, ERM210 treatment significantly enhanced cellular apoptosis in a dose- and timedependent manner in HepG2 cells. To understand the effect of ERM210 on apoptosis-related protein expression, HepG2 cells were treated with ERM210 at a concentration of $15 \%$ for $0,1,3,6,12$, and $24 \mathrm{~h}$. The levels of apoptosis-related proteins were determined using western blot assay. The obtained results showed that after treating HepG2 cells with ERM210, the expression of pro-apoptotic proteins, such as cleaved caspase-3, -7 and -9 , and BAX, was significantly up- 
A

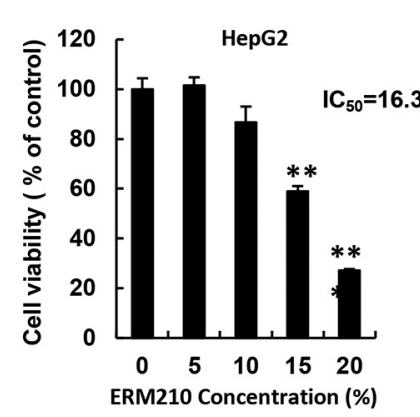

C

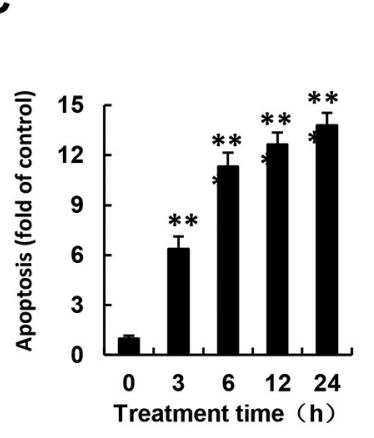

D

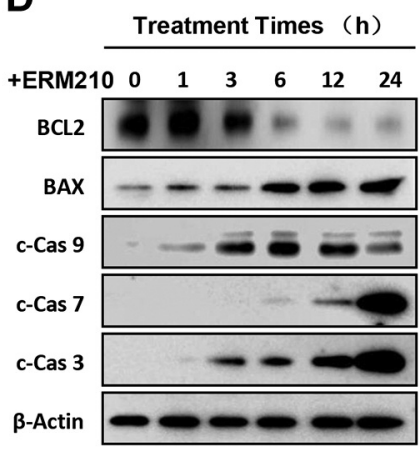

B
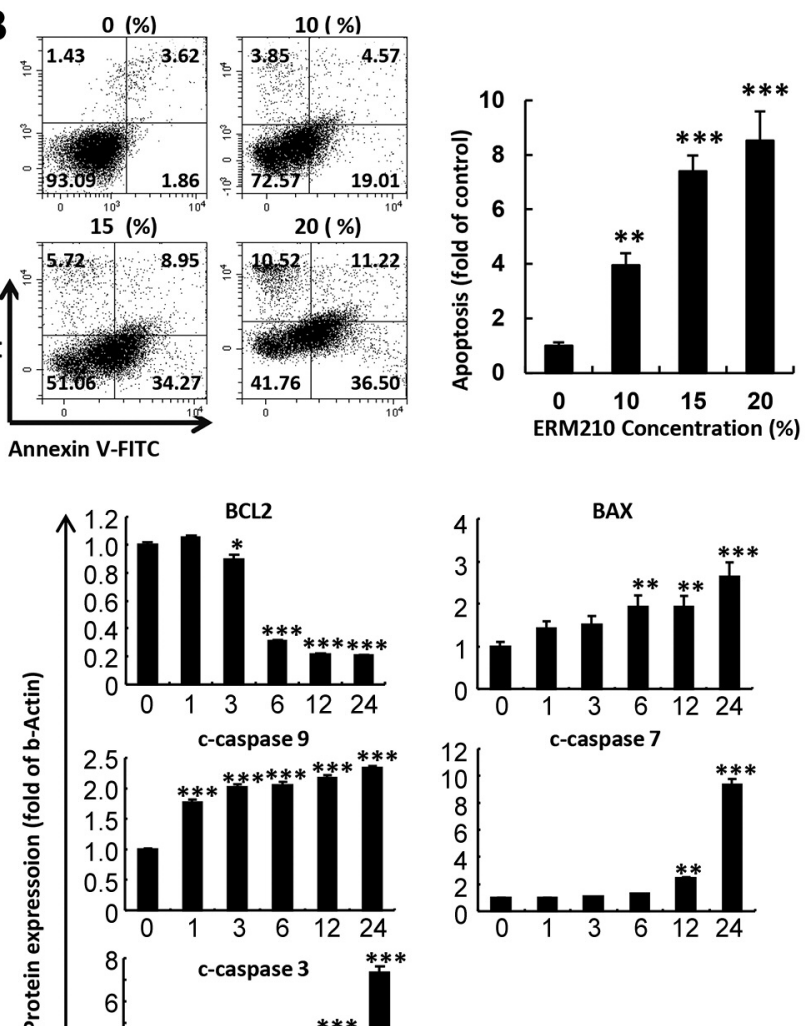
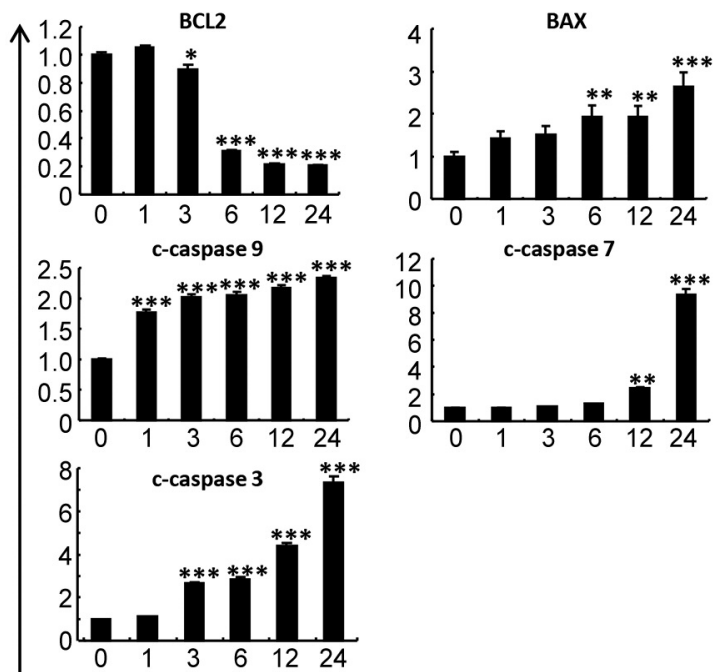

Treatment time (h)

Figure 1. ERM210 reduces cell viability and induces concentration-dependent apoptosis of HepG2 cells. A: HepG2 liver cancer cells and QSG7701 normal liver cells were treated with ERM210, following which their cell viability was evaluated by 3-(4,5-dimethylthiazol-2-yl)-2,5diphenyltetrazolium bromide assay. B: Concentration-dependent apoptosis was determined in HepG2 cells treated with ERM210 for 24 h, after which annexin V-fluorescein isothiocyanate (FITC) and propidium iodide (PI) staining was performed for 25 min followed by flow cytometry. A representative cytogram and quantitative analysis are shown. C: HepG2 cells were treated with $15 \%$ ERM210 for different times followed by annexin V-FITC staining. Apoptosis of HepG2 cells was quantified using flow cytometry. D: Western blotting was used to detect pro-apoptotic BCL2associated X, apoptosis regulator (BAX), cleared caspases (c-Cas), and anti-apoptotic proteins BCL2 apoptosis regulator (BCL2) in HepG2 cells treated with ERM210 for different times. Proteins were quantitatively normalized by $\beta$-actin protein. A representative blot and quantitative analysis are shown. Significantly different at: $* p<0.05, * * p<0.01$, and $* * *<<0.001$.

regulated; conversely, the expression of anti-apoptotic BCL2 protein was down-regulated (Figure 1D).

ERM210 induced the accumulation of ROS, inhibiting HepG2 cell migration. To determine the effect of ERM210 on intracellular and mitochondrial ROS, HepG2 cells were treated with $0,10,15$, and $20 \%$ ERM210 for $24 \mathrm{~h}$. The cells were stained with DHE (whole-cell ROS detector) and MitoSOX (a detector of mitochondrial ROS) and observed using fluorescence microscopy and flow cytometry. The results showed that after treatment of HepG2 cells with different concentrations of ERM210 $(0,10,15$, and $20 \%)$ for $24 \mathrm{~h}$, the intracellular and mitochondrial ROS levels significantly increased in a dose-dependent manner (Figure $2 \mathrm{~A}$ and $\mathrm{B}$ ). In addition, to determine the effects of ERM210 on cellmigration ability, the wound-healing assay was also performed after ERM210 treatment of HepG2 cells. As shown in Figure $2 \mathrm{C}$ and D, ERM210 treatment significantly inhibited cell migration of HepG2 cells by down-regulating the expression of vimentin protein (a marker of cell migration).

ROS were found to play a crucial role in ERM210-induced cell apoptosis and migration of HepG2 cells. To verify that ROS is the principal factor modulating ERM210-induced cell apoptosis, HepG2 cells were pre-treated with NAC for $30 \mathrm{~min}$ and then treated with ERM210 (15\%) for $24 \mathrm{~h}$. The 
A
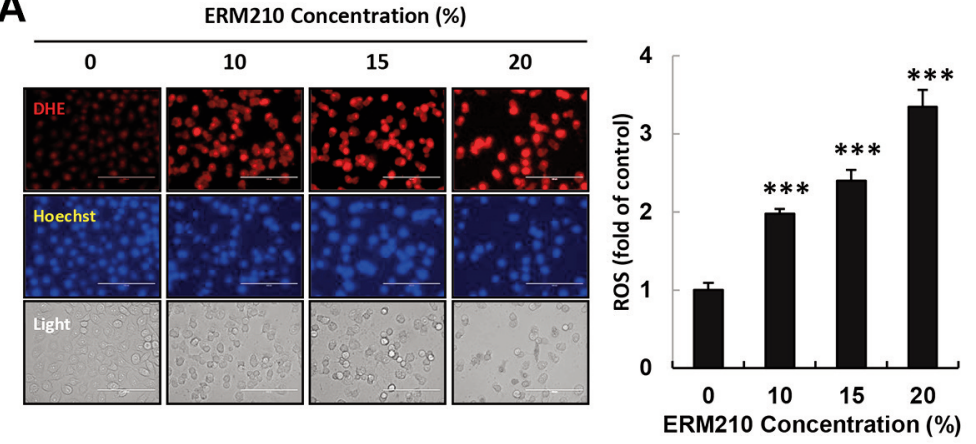

B

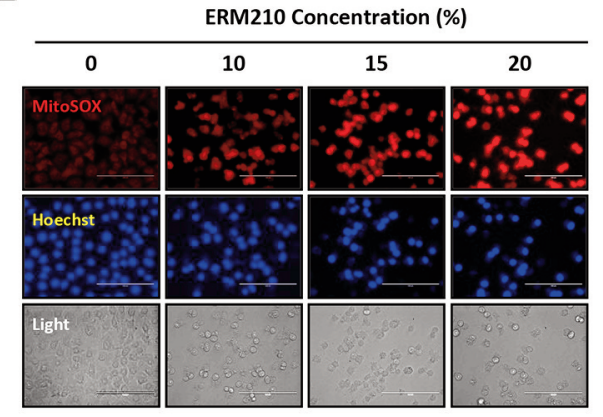

C
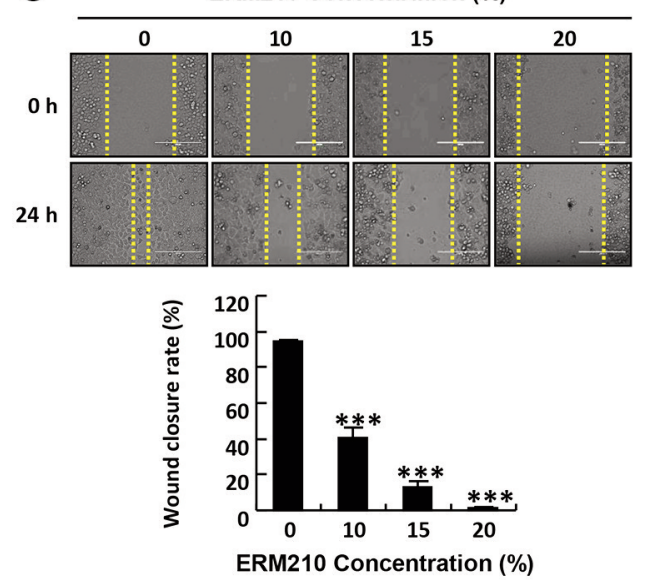

D

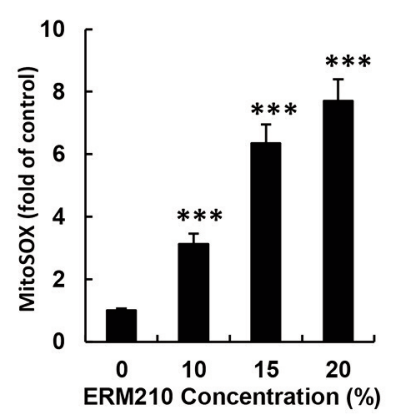

ERM210

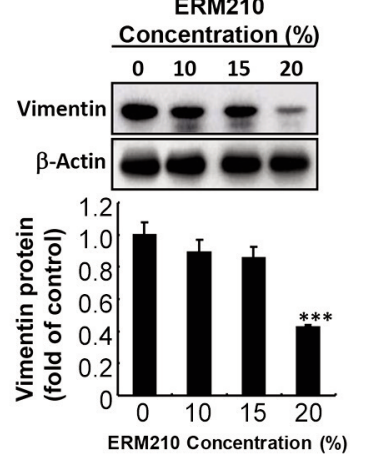

Figure 2. ERM210 induced the accumulation of reactive oxygen species (ROS) and reduced the migration of HepG2 cells. Cells were treated with different concentrations of ERM210 for $24 \mathrm{~h}$. A: Intracellular ROS levels were determined by staining with dihydroethidium and Hoechst using fluorescence microscopy (left) and flow cytometry (right). B: Mitochondrial ROS levels in HepG2 cells were detected by MitoSOX staining and fluorescence microscopy (left) and flow cytometry (right). C: Wound-healing assay examined the migration ability of HepG2 cells. A representative image and quantitative analysis are shown. D: The expression of the migration marker vimentin was analyzed by western blot. A representative blot and quantitative analysis are shown. Significantly different at $* * * p<0.001$.

intracellular and mitochondrial ROS levels were determined by staining with DHE and MitoSOX. The obtained results indicated that NAC pre-treatment significantly reduced the intracellular (Figure 3A) and mitochondrial (Figure 3B) ROS levels in HepG2 cells. We simultaneously examined the effect of NAC on cell migration and apoptosis upon ERM210 stimulation. As shown in Figure 3C and D, NAC treatment significantly suppressed ERM210-induced cell migration and apoptosis, delineating the important regulatory role of ERM210 on ROS. Compared with untreated HepG2 cells pretreated with NAC alone as a control, there was no difference in the intracellular ROS levels of the two groups (data not shown).

ERM210 induced apoptosis of HepG2 cells via MAPK signaling pathways. To clarify the molecular mechanisms underlying the effects of ROS on ERM210-induced apoptosis and cell migration, we also analyzed the expression of apoptosis- and migration-related proteins following NAC treatment. The obtained results showed that the addition of NAC reduced the expression of cleaved caspase-3, -7 and -9 , and of BAX (pro-apoptotic) and vimentin (migration marker) proteins in HepG2 cells induced by ERM210 and restored BCL2 (anti-apoptotic) protein expression (Figure 4A). In order to exclude the influence of NAC on cells during the ERM210-induced initiation of the MAPK signaling pathway of HepG2 cells, we set up a separate NAC treatment group which showed that treatment with NAC alone had no effect on cells (data not shown). Furthermore, the effect of ERM210 on ROS-dependent MAPK signaling pathways was also detected by the western blot assay. As shown in Figure 4B, after treating HepG2 cells with ERM210, p38 and ERK protein phosphorylation was significantly up-regulated while that of JNK phosphorylation was down-regulated. 
A

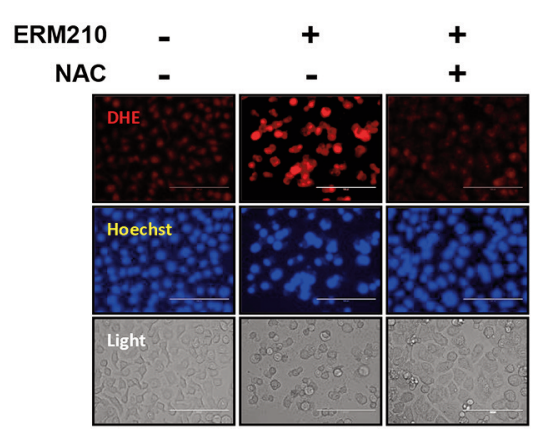

B

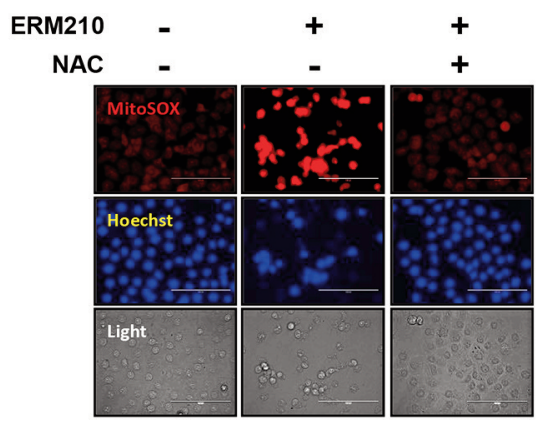

C

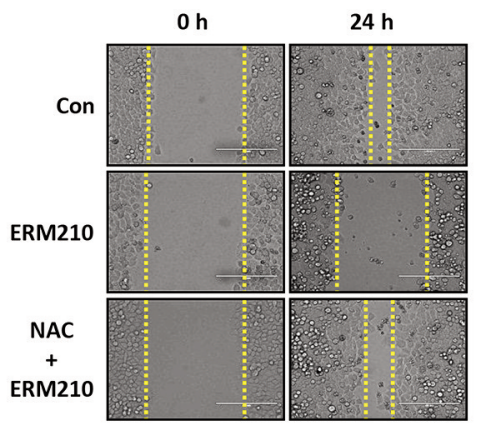

D
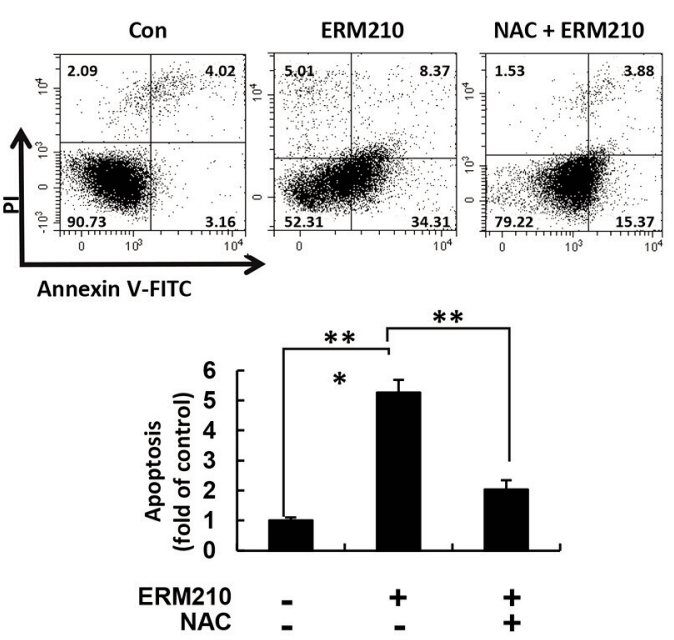

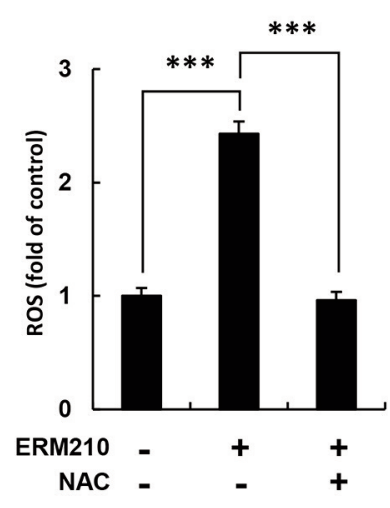

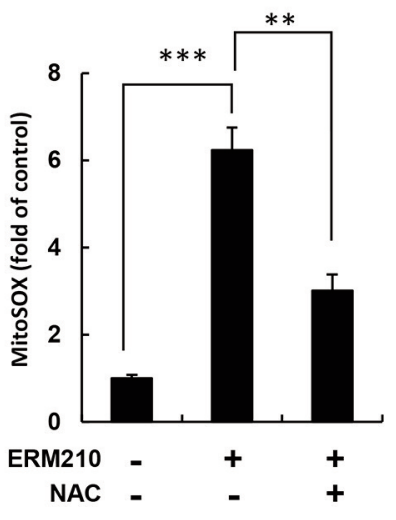

Figure 3. Inhibition of intracellular reactive oxygen species (ROS) reduced cell apoptosis and migration of HepG2 cells. Cells were pre-treated with reactive oxygen species scavenger $N$-acetylcysteine (NAC) followed by treatment with $15 \%$ ERM 210 for 24 h. HepG 2 cells were stained with dihydroethidium, MitoSOX, and Hoechst. They were then subjected to fluorescence microscopy (upper panels) and flow cytometry (lower panels) to demonstrate the impact on the cellular (A) and mitochondrial (B) ROS levels in HepG2 cells. Representative images and quantitative analyses are shown. C: Wound-healing assays examined the ability of HepG2 cells to migrate after pre-treatment with NAC then ERM210. D: After pretreating HepG2 cells with NAC, the apoptotic effect of ERM210 on HepG2 cells was detected by flow cytometry. Significantly different at: *p<0.01, and $* * * p<0.001$.

\section{Discussion}

In recent years, oxidative stress has been found to play a pivotal role in the progression of several cancer types. Mitochondria are the main source of ROS, large amounts of which are generated within the electron transport chain (24). The excessive production of ROS leads to intracellular mitochondrial damage (such as a reduction of mitochondrial membrane potential, increased mitochondrial ROS and changes in the morphology of the mitochondrial ridge), which has emerged as a major focus area for current cancer treatment (25-27). Therefore, ROS play an indispensable role in cell growth, differentiation, metabolism, and proliferation.

The occurrence of liver cancer is mostly attributable to infection with hepatitis B virus, liver cirrhosis, alcoholism, smoking, and other detrimental habits (28). Studies have shown that Asian traditional herbal medicine exerts antiinflammatory $(29,30)$, antibacterial (31), antitumor (32-34), and other physiological actions (35-37). Its antitumor effect is relatively significant. Asian traditional herbal medicine has demonstrated potent anticancer activity, with low toxicity and minimal side-effects; the prognosis of patients is favorable, and patient lifespan is enhanced [reviewed in (38)], Therefore, we further explored the potential of Asian traditional herbal medicines as an innovative avenue for using novel therapeutic drugs in liver cancer treatment.

In the present study, we showed that ERM210, composed of a variety of Chinese herbal medicines, induced apoptosis of HepG2 liver cancer cells. Since ERM210 contains a variety of components from Asian traditional herbal 
A

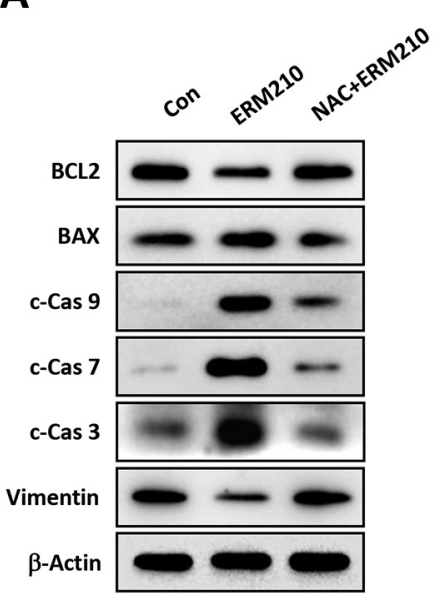

B

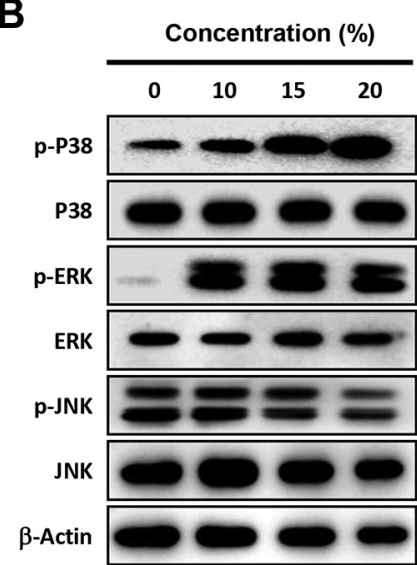

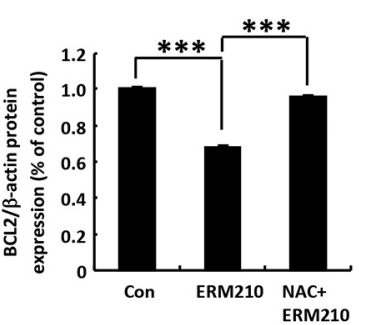
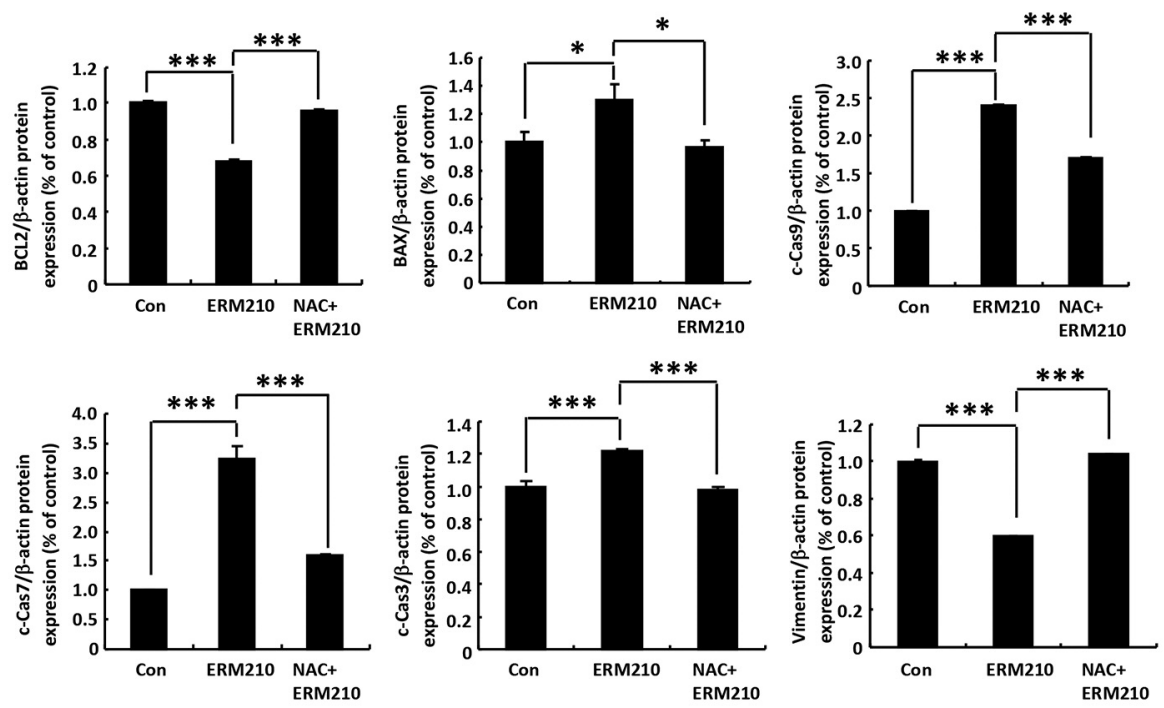

Figure 4. ERM210 induced apoptosis via mitogen-activated protein kinase (MAPK) signaling pathways in HepG2 cells. A: Western blot assays were used to detect the apoptotic effect of ERM210 on HepG2 cells. HepG2 cells were pretreated with reactive oxygen species scavenger $N$-acetylcysteine (NAC) for $30 \mathrm{~min}$ and then treated with ERM210 for $24 \mathrm{~h}$. The expression of apoptosis-related proteins namely cleaved caspases (c-Cas), BCL2 apoptosis regulator (BCL2), BCL2-associated X apoptosis regulator $(B A X)$, and the migration-related protein vimentin were analyzed by western blotting (left) and quantified (right). B: The levels of native and phosphorylated (p) p38, extracellular-regulated kinase (ERK), and c-Jun N-terminal kinase (JNK) proteins in the MAPK signaling pathway were determined after treating HepG2 cells with ERM210 for 24 h by western blotting (left) and quantified (right). Significantly different at: $* p<0.05$, and $* * * p<0.001$.

medicine, all of its main components have anticancer effects and may have toxic and side-effects on normal cells ERM210 was significantly more toxic towards HepG2 cells than QSG-7701 cells (normal liver cells). We speculate that the reason for this may be that ERM210 has a certain targeting effect on liver cancer cells.

We optimized effective ingredients into ERM210 with the aim of producing a Chinese herbal medicine that is more effective on cancer cells and has lower toxic and side-effects on normal cells to achieve better therapeutic effects. We found that ERM210 induced a rise in the ROS level and caused mitochondrial dysfunction in HepG2 cells treated with ERM210, which in turn triggered cell apoptosis. When
NAC (ROS scavenger) was added, there was a steep decline in the intracellular and mitochondrial ROS leveIs, and cell apoptosis reduced significantly. These results indicate that ERM210 induces HepG2 cell apoptosis by facilitating an increase of ROS in HepG2 cells. The MAPK signaling pathway plays an important role in cells, controlling a series of physiological activities such as cell growth, differentiation, and proliferation (39). Numerous studies have shown that MAPKs play an indispensable role in the regulation of cell physiological activity by ROS (40-42). The results of the present study showed that the MAPK signaling pathway is particularly important in the process of ERM210induced apoptosis of HepG2 cells. 
Apoptosis can be classified into the endogenous and exogenous pathways (43). When a cell is externally stimulated, mitochondrial damage within the cell releases cytochrome $c$ and activates caspase-9. The cleavage of caspase- 9 in turn cleaves caspase- 3 and induces cell apoptosis (44). In the process of cell apoptosis, the BCL2 family is critical because it regulates various physiological activities of mitochondria in cells [reviewed in (45)]. The BCL2 family is divided into anti-apoptotic and pro-apoptotic proteins. When mitochondria are damaged, the levels of anti-apoptotic proteins reduces while there is a rise in the pro-apoptotic protein level. This is accompanied by a significant increase in the expression of cleaved caspase-3. Our study provides confirmation that ROS are key to ERM210-induced apoptosis of HepG2 cells as after pretreatment of the cell with NAC, intracellular and mitochondria ROS were significantly reduced in HepG2 cells. ERM210-induced cell apoptosis was also significantly reduced by the addition of NAC, with a concomitant increase in the expression of anti-apoptotic BCL2 and reduction in the expression of pro-apoptotic BAX. Additionally, the extent of mitochondrial damage, as well as the cleavage of caspases 3,7 , and 9 proteins was significantly reduced. These results all indicated that ROS may be a key factor in ERM210-induced apoptosis of HepG2 cells.

With our results, we demonstrate that the MAPK signaling pathway is considerably altered during ERM210-induced apoptosis of HepG2 cells, and plays an important role. The increase in cell survival rate upon inhibition of the intracellular and mitochondrial ROS further reiterates the important role of ROS in the process of ERM210-induced apoptosis.

ERM210 may also induce apoptosis of liver cancer cells through other methods such as changes in cell metabolism, cell membrane composition or other signaling pathways. However, since ERM210 has not yet been analyzed by liquid chromatography, the precise compounds responsible for such effects remain unknown. Nevertheless, we demonstrate that ERM210 indeed induces apoptosis of HepG2 cells through a ROS-mediated mechanism. Our results, therefore, establish the prospect of developing ERM210 as a new therapeutic drug for clinically treating liver cancer and lays a suitable foundation for the development of subsequent liver cancer treatment approaches in future studies.

\section{Conflicts of Interest}

The Authors declare that they have no competing interests in regard to this study.

\section{Authors' Contributions}

Conceptualization: JL, YXG, DPX, HNS and TK. Methodology: HNS and TK. Software: TK and HNS. Validation: TK and HNS.
Formal analysis: JL, YXG, DPX, HJ, HS, JK, YHP, HNS and TK. Investigation: JL, YXG, DPX, HNS and TK. Resources: TK and HNS. Data curation: TK and HNS. Writing - original draft: JL, YXG, DPX, HNS and TK. Writing - review \& editing: TK and HNS. Visualization: TK and HNS. Supervision: TK and HNS. Project administration: TK and HNS. Funding acquisition: TK. All Authors read and approved the final article.

\section{Acknowledgements}

The present study was supported by Basic Science Research Program through the National Research Foundation of Korea (NRF) funded by the Ministry of Education (2020R1I1A2052417) and The Korean Research Institute of Bioscience and Biotechnology Research Information System (RBM0112112).

\section{References}

1 Tu Y, Zhu S, Wang J, Burstein E and Jia D: Natural compounds in the chemoprevention of alcoholic liver disease. Phytother Res 33(9): 2192-2212, 2019. PMID: 31264302. DOI: 10.1002/ptr.6410

2 Kong LZ, Chandimali N, Han YH, Lee DH, Kim JS, Kim SU, Kim TD, Jeong DK, Sun HN, Lee DS and Kwon T: Pathogenesis, early diagnosis, and therapeutic management of alcoholic liver disease. Int J Mol Sci 20(11): 2712, 2019. PMID: 31159489. DOI: 10.3390/ijms20112712

3 Singh A and Zhao K: Treatment of insomnia with traditional Chinese herbal medicine. Int Rev Neurobiol 135: 97-115, 2017. PMID: 28807167. DOI: 10.1016/bs.irn.2017.02.006

4 Rehman SU, Choe K and Yoo HH: Review on a traditional herbal medicine, Eurycoma longifolia Jack (Tongkat Ali): Its traditional uses, chemistry, evidence-based pharmacology and toxicology. Molecules 21(3): 331, 2016. PMID: 26978330. DOI: $10.3390 /$ molecules 21030331

5 Howes MR, Fang R and Houghton PJ: Effect of Chinese herbal medicine on Alzheimer's disease. Int Rev Neurobiol 135: 29-56, 2017. PMID: 28807163. DOI: 10.1016/bs.irn.2017.02.003

6 Liu C and Huang Y: Chinese herbal medicine on cardiovascular diseases and the mechanisms of action. Front Pharmacol 7: 469, 2016. PMID: 27990122. DOI: 10.3389/fphar.2016.00469

7 Ong M, Peng J, Jin X and Qu X: Chinese herbal medicine for the optimal management of polycystic ovary syndrome. Am J Chin Med 45(3): 405-422, 2017. PMID: 28359195. DOI: 10.1142/S0192415X17500252

8 Chen X, Deng L, Jiang X and Wu T: Chinese herbal medicine for oesophageal cancer. Cochrane Database Syst Rev (1): CD004520, 2016. PMID: 26799001. DOI: 10.1002/14651858. CD004520.pub7

9 Zhang XW, Liu W, Jiang HL and Mao B: Chinese herbal medicine for advanced non-small-cell lung cancer: a systematic review and meta-analysis. Am J Chin Med 46(5): 923-952, 2018. PMID: 30001642. DOI: 10.1142/S0192415X18500490

10 Luo H, Vong CT, Chen H, Gao Y, Lyu P, Qiu L, Zhao M, Liu Q, Cheng Z, Zou J, Yao P, Gao C, Wei J, Ung COL, Wang S, Zhong $\mathrm{Z}$ and Wang Y: Naturally occurring anti-cancer compounds: shining from Chinese herbal medicine. Chin Med 14: 48, 2019. PMID: 31719837. DOI: 10.1186/s13020-019-0270-9

11 Yeung KS, Hernandez M, Mao JJ, Haviland I and Gubili J: Herbal medicine for depression and anxiety: A systematic review 
with assessment of potential psycho-oncologic relevance. Phytother Res 32(5): 865-891, 2018. PMID: 29464801. DOI: $10.1002 /$ ptr.6033

12 Kalyanaraman B, Cheng G, Hardy M, Ouari O, Bennett B and Zielonka J: Teaching the basics of reactive oxygen species and their relevance to cancer biology: Mitochondrial reactive oxygen species detection, redox signaling, and targeted therapies. Redox Biol 15: 347-362, 2018. PMID: 29306792. DOI: 10.1016/ j.redox.2017.12.012

13 Yang Y, Karakhanova S, Hartwig W, D'Haese JG, Philippov PP, Werner J and Bazhin AV: Mitochondria and mitochondrial ROS in cancer: Novel targets for anticancer therapy. J Cell Physiol 231(12): 2570-2581, 2016. PMID: 26895995. DOI: 10.1002/ jcp. 25349

14 Kajarabille N and Latunde-Dada GO: Programmed cell-death by ferroptosis: Antioxidants as mitigators. Int J Mol Sci 20(19): 4968, 2019. PMID: 31597407. DOI: 10.3390/ijms20194968

15 Xie DP, Gong YX, Jin YH, Ren CX, Liu Y, Han YH, Jin MH, Zhu D, Pan QZ, Yu LY, Lee DS, Lee J, Kim J, Park YH, Hyun JW, Kwon T, Cui YD and Sun HN: Anti-tumor properties of Picrasma quassioides extracts in $\mathrm{H}-\mathrm{Ras} \mathrm{G} 12 \mathrm{~V}$ liver cancer are mediated through ROS-dependent mitochondrial dysfunction. Anticancer Res 40(7): 3819-3830, 2020. PMID: 32620621. DOI: 10.21873/anticanres. 14371

16 Nissanka N and Moraes CT: Mitochondrial DNA damage and reactive oxygen species in neurodegenerative disease. FEBS Lett 592(5): 728-742, 2018. PMID: 29281123. DOI: 10.1002/18733468.12956

17 Qian Q, Chen W, Cao Y, Cao Q, Cui Y, Li Y and Wu J: Targeting reactive oxygen species in cancer via Chinese herbal medicine. Oxid Med Cell Longev 2019: 9240426, 2019. PMID: 31583051. DOI: $10.1155 / 2019 / 9240426$

18 Phang CW, Karsani SA, Sethi G and Abd Malek SN: Flavokawain $\mathrm{C}$ inhibits cell cycle and promotes apoptosis, associated with endoplasmic reticulum stress and regulation of MAPKs and Akt signaling pathways in HCT 116 human colon carcinoma cells. PLoS One 11(2): e0148775, 2016. PMID: 26859847. DOI: 10.1371/journal.pone.0148775

19 Yue J and López JM: Understanding MAPK signaling pathways in apoptosis. Int J Mol Sci 21(7): 2346, 2020. PMID: 32231094. DOI: 10.3390/ijms21072346

20 Wang C, Li P, Xuan J, Zhu C, Liu J, Shan L, Du Q, Ren Y and Ye J: Cholesterol enhances colorectal cancer progression via ROS elevation and MAPK signaling pathway activation. Cell Physiol Biochem 42(2): 729-742, 2017. PMID: 28618417. DOI: $10.1159 / 000477890$

$21 \mathrm{Xu} \mathrm{H}$, Zhao X, Liu X, Xu P, Zhang K and Lin X: Antitumor effects of traditional Chinese medicine targeting the cellular apoptotic pathway. Drug Des Devel Ther 9: 2735-2744, 2015. PMID: 26056434. DOI: 10.2147/DDDT.S80902

22 Wang Z, Qi F, Cui Y, Zhao L, Sun X, Tang W and Cai P: An update on Chinese herbal medicines as adjuvant treatment of anticancer therapeutics. Biosci Trends 12(3): 220-239, 2018. PMID: 30012913. DOI: 10.5582/bst.2018.01144

23 Qi F, Li A, Inagaki Y, Gao J, Li J, Kokudo N, Li XK and Tang W: Chinese herbal medicines as adjuvant treatment during chemo- or radio-therapy for cancer. Biosci Trends 4(6): 297-307, 2010. PMID: 21248427.

24 Kleih M, Böpple K, Dong M, Gaißler A, Heine S, Olayioye MA, Aulitzky WE and Essmann F: Direct impact of cisplatin on mitochondria induces ROS production that dictates cell fate of ovarian cancer cells. Cell Death Dis 10(11): 851, 2019. PMID: 31699970. DOI: 10.1038/s41419-019-2081-4

25 Raza MH, Siraj S, Arshad A, Waheed U, Aldakheel F, Alduraywish S and Arshad M: ROS-modulated therapeutic approaches in cancer treatment. J Cancer Res Clin Oncol 143(9): 1789-1809, 2017. PMID: 28647857. DOI: 10.1007/s00432-0172464-9

26 Storz P: Reactive oxygen species in tumor progression. Front Biosci 10: 1881-1896, 2005. PMID: 15769673. DOI: 10.2741/ 1667

27 Ismail T, Kim Y, Lee H, Lee DS and Lee HS: Interplay between mitochondrial peroxiredoxins and ROS in cancer development and progression. Int J Mol Sci 20(18): 4407, 2019. PMID: 31500275. DOI: 10.3390/ijms20184407

28 Orcutt ST and Anaya DA: Liver resection and surgical strategies for management of primary liver cancer. Cancer Control 25(1): 1073274817744621, 2018. PMID: 29327594. DOI: 10.1177/ 1073274817744621

29 Runfeng L, Yunlong H, Jicheng H, Weiqi P, Qinhai M, Yongxia S, Chufang L, Jin Z, Zhenhua J, Haiming J, Kui Z, Shuxiang H, Jun D, Xiaobo L, Xiaotao H, Lin W, Nanshan Z and Zifeng Y: Lianhuaqingwen exerts anti-viral and anti-inflammatory activity against novel coronavirus (SARS-CoV-2). Pharmacol Res 156: 104761, 2020. PMID: 32205232. DOI: 10.1016/j.phrs.2020.104761

30 Li SY, Zhou YL, He DH, Liu W, Fan XZ, Wang Q, Pan HF, Cheng YX and Liu YQ: Centipeda minima extract exerts antineuroinflammatory effects via the inhibition of NF-kB signaling pathway. Phytomedicine 67: 153164, 2020. PMID: 31954258. DOI: 10.1016/j.phymed.2019.153164

31 Yamada T, Wajima T, Nakaminami H, Kobayashi K, Ikoshi H and Noguchi N: The modified Gingyo-san, a Chinese herbal medicine, has direct antibacterial effects against respiratory pathogens. BMC Complement Altern Med 16(1): 463, 2016. PMID: 27842538. DOI: 10.1186/s12906-016-1431-3

32 Chen F, Zhong Z, Tan HY, Guo W, Zhang C, Tan CW, Li S, Wang $\mathrm{N}$ and Feng Y: Uncovering the anticancer mechanisms of Chinese herbal medicine formulas: Therapeutic alternatives for liver cancer. Front Pharmacol 11: 293, 2020. PMID: 32256363. DOI: $10.3389 /$ fphar.2020.00293

33 Qi L, Zhang Y, Song F and Ding Y: Chinese herbal medicine promote tissue differentiation in colorectal cancer by activating HSD11B2. Arch Biochem Biophys 695: 108644, 2020. PMID: 33098869. DOI: 10.1016/j.abb.2020.108644

34 Huang WC, Chang MS, Huang SY, Tsai CJ, Kuo PH, Chang HW, Huang ST, Kuo CL, Lee SL and Kao MC: Chinese herbal medicine Ganoderma tsugae displays potential anti-cancer efficacy on metastatic prostate cancer cells. Int J Mol Sci 20(18): 4418, 2019. PMID: 31500366. DOI: 10.3390/ijms20184418

35 Akalın E, Ekici M, Alan Z, Özbir Elevli E, Yaman Bucak A, Aobuliaikemu $\mathrm{N}$ and Üresin AY: Traditional Chinese medicine practices used in COVID-19 (Sars-cov 2/Coronavirus-19) treatment in clinic and their effects on the cardiovascular system. Turk Kardiyol Dern Ars 48(4): 410-424, 2020. PMID: 32519978. DOI: $10.5543 /$ tkda.2020.03374

36 Liu C, Li S, Zhang Q, Guo F, Tong M, Martinez MFYM, Wang $\mathrm{HH}$, Zhao Y and Shang D: Emerging role of Chinese herbal medicines in the treatment of pancreatic fibrosis. Am J Chin Med 47(4): 709-726, 2019. PMID: 31091974. DOI: 10.1142/ S0192415X1950037X 
37 Zhang S, Liu X, Sun M, Zhang Q, Li T, Li X, Xu J, Zhao X, Chen D and Feng X: Reversal of reserpine-induced depression and cognitive disorder in zebrafish by sertraline and Traditional Chinese Medicine (TCM). Behav Brain Funct 14(1): 13, 2018. PMID: 29898741. DOI: 10.1186/s12993-018-0145-8

38 Hsiao WL and Liu L: The role of traditional Chinese herbal medicines in cancer therapy - from TCM theory to mechanistic insights. Planta Med 76(11): 1118-1131, 2010. PMID: 20635308. DOI: $10.1055 / \mathrm{s}-0030-1250186$

39 Lee Y, Kim YJ, Kim MH and Kwak JM: MAPK cascades in guard cell signal transduction. Front Plant Sci 7: 80, 2016. PMID: 26904052. DOI: 10.3389/fpls.2016.00080

40 Gong L, Lei Y, Liu Y, Tan F, Li S, Wang X, Xu M, Cai W, Du B, Xu F, Zhou Y, Han H, Sun H and Qiu L: Vaccarin prevents ox-LDL-induced HUVEC EndMT, inflammation and apoptosis by suppressing ROS/p38 MAPK signaling. Am J Transl Res 11(4): 2140-2154, 2019. PMID: 31105824.

41 Su X, Shen Z, Yang Q, Sui F, Pu J, Ma J, Ma S, Yao D, Ji M and Hou P: Vitamin C kills thyroid cancer cells through ROSdependent inhibition of MAPK/ERK and PI3K/AKT pathways via distinct mechanisms. Theranostics 9(15): 4461-4473, 2019. PMID: 31285773. DOI: 10.7150/thno.35219
42 Schattauer SS, Bedini A, Summers F, Reilly-Treat A, Andrews MM, Land BB and Chavkin C: Reactive oxygen species (ROS) generation is stimulated by $\mathrm{k}$ opioid receptor activation through phosphorylated c-Jun N-terminal kinase and inhibited by $\mathrm{p} 38$ mitogen-activated protein kinase (MAPK) activation. J Biol Chem 294(45): 16884-16896, 2019. PMID: 31575661. DOI: 10.1074/jbc.RA119.009592

$43 \mathrm{Xu}$ X, Lai Y and Hua ZC: Apoptosis and apoptotic body: disease message and therapeutic target potentials. Biosci Rep 39(1): BSR20180992, 2019. PMID: 30530866. DOI: 10.1042/ BSR20180992

44 Brunelle JK and Letai A: Control of mitochondrial apoptosis by the Bcl-2 family. J Cell Sci 122(Pt 4): 437-441, 2009. PMID: 19193868. DOI: $10.1242 /$ jcs.031682

45 Youle RJ and Strasser A: The BCL-2 protein family: opposing activities that mediate cell death. Nat Rev Mol Cell Biol 9(1): 47-59, 2008. PMID: 18097445. DOI: 10.1038/nrm2308

Received May 3, 2021

Revised May 29, 2021

Accepted June 1, 2021 